Pacific Journal of Mathematics

THE CONVEX GENERATION OF CONVEX BOREL SETS IN 


\section{THE CONVEX GENERATION OF CONVEX BOREL SETS IN EUCLIDEAN SPACE}

\section{A. Rogers}

The main object of this note is to resolve a problem of V. L. Klee on the convex generation of convex Borel sets in Euclidean space, into a sequence of three problems, each of some intrinsic interest; the joint solution of the three problems being equivalent to the solution of Klee's problem.

In general terms, Klee asks the question whether a convex Borel set can be generated by the Borel method from the closed (or open) convex sets without at any stage leaving the domain of convex sets. More specifically he calls a set $K$ a convexly generated Borel set if:

A. $K$ belongs to the minimal system of sets, containing the closed convex sets, that is closed under the operations of countable increasing union and of countable decreasing intersection.

Naturally the operations of countable increasing union and of countable decreasing intersection lead from the class of convex sets to the class of convex sets. Klee asks whether the class of convexly generated Borel sets coincides with the class of convex sets that are Borel sets. D. G. Larman [6] has recently given the answer 'yes' in 3-dimensional space, using methods that do not readily generalize.

It is not quite clear why Klee works with condition $A$. It might seem equally reasonable to work with the sets $K$ satisfying the condition that:

$B$. $K$ belongs to the minimal system of sets, containing the closed convex sets, that is closed under the operations of countable increasing union and of countable intersection.

Here of course it is the penultimate word of $A$ that has been omitted. D. G. Larman [7] has shown that these two conditions are equivalent.

But there are other operations that lead from convex Borel sets to convex Borel sets. We show, in $\S 2$ below, that the smallest convex set containing two convex Borel sets is a convex Borel set, by a method that seems to depend essentially on the properties of Euclidean space. This result is perhaps a little surprising in view of the facts that the smallest convex set containing a Borel set is not necessarily a Borel set (even in $E_{3}$ ) and that the vector sum of two Borel sets on the line is not necessarily a Borel set (see [8] and [1]).

Thus it is reasonable to call a set $K$ a weakly convexly generated Borel set if it satisfies the condition: 
C. $K$ belongs to the minimal system of sets, containing the closed convex sets, that is closed under the operations of taking the least convex cover of two sets of the system, of countable increasing union and of countable intersection.

It is easy to verify (see $\S 3$ below) that this system of weakly convexly generated Borel sets coincides with the system of sets $K$ satisfying the apparently weaker condition:

$D$. $K$ belongs to the minimal system of sets, containing the closed convex sets, that is closed under the operations of taking the least convex cover of any countable union, and of taking any countable intersection.

In some ways, the Souslin operation is a more convenient way of generating Borel sets than the transfinite Borel process, but of course, in general, it leads to sets that are not necessarily Borel sets. But the Borel sets are the Souslin sets that have Borel-Souslin representations (see [9]). This suggests that we introduce the sets with a convex-Borel-Souslin representation. A set $K$ will be said to have such a representation if it satisfies the condition:

$E$. $K$ can be represented in the form

$$
K=\bigcup_{i_{1}, i_{2}}, \ldots \bigcap_{n=1}^{\infty} F\left(i_{1}, i_{2}, \cdots, i_{n}\right),
$$

where the union is taken over all infinite sequences $i_{1}, i_{2}, \ldots$ of positive integers, where the sets

$$
F\left(i_{1}, i_{2}, \cdots, i_{n}\right)
$$

are compact convex sets for all finite sequences $i_{1}, i_{2}, \cdots, i_{n}$ of positive integers, and where the sets

$$
\begin{aligned}
K\left(i_{1}, i_{2}, \cdots i_{n}\right)= & \bigcup_{j_{n+1}, j_{n+2}, \cdots}\left[\left\{\bigcap_{r=1}^{n} F\left(i_{1}, i_{2}, \cdots, i_{r}\right)\right\}_{n}\right. \\
& \left.\left\{\bigcap_{r=n+1}^{\infty} F\left(i_{1}, i_{2}, \cdots, i_{n}, j_{n+1}, j_{n+2}, \cdots, j_{r}\right)\right\}\right],
\end{aligned}
$$

are convex Borel sets, for all finite sequences $i_{1}, i_{2}, \cdots, i_{n}$ of positive integers, including the sequence of zero length.

We prove, in $\S 4$ below, that the weakly convexly generated Borel sets all have such convex-Borel-Souslin representations.

It is convenient to introduce a final condition:

$F$. $K$ is a convex Borel set.

In terms of these conditions our results take the form

$$
A \Leftrightarrow B \Rightarrow C \Leftrightarrow D \Rightarrow E \Rightarrow F .
$$

the main results being the justification of $C$ as relevent, by showing 
that the least convex set containing the union of two convex Borel sets is a Borel set, and the proof that $D \Rightarrow E$.

In these terms, Klee's conjecture takes the form

$$
F \Longrightarrow A \text {. }
$$

This is clearly equivalent to the conjunction of the three conjectured implications

$$
F \Rightarrow E, \quad E \Rightarrow D, \quad C \Rightarrow B
$$

These conjectures seem to me to be of interest in their own right; especially as Larman's result implies that they are true in 3-dimensional space. It seems unlikely that none can be extended to general Euclidean space.

This approach suggests a further conjecture analogous to Klee's conjecture. It seems possible that every convex Souslin set has a convex-Souslin representation; i.e. a representation of the type described in condition $E$ without the requirement that the sets $K\left(i_{1}, i_{2}\right.$, $\cdots, i_{n}$ ) be Borel sets. A proof of this might be a valuable first step towards the proof that $F \Rightarrow E$.

2. The convex set containing two convex Borel sets. Our first result is an immediate consequence of the Kunugui-Novikoff theorem, which states that, if a Borel set $B$ in $E_{n}$ has the property that all sections by the lines of the form

$$
x_{1}=a_{1}, \quad x_{2}=a_{2}, \cdots, \quad x_{n-1}=a_{n-1},
$$

are $\mathscr{F}_{\sigma}$-sets, then the orthogonal projection of $B$ on the plane, with equation

$$
x_{n}=0 \text {, }
$$

is a Borel set. This result appears in a paper of Kunugui [4]; but D. G. Larman (private communication) has drawn my attention to a flaw in Kunugui's paper. A very complicated proof, based on unpublished work of Novikoff is given in [5].

If $B$ is a convex Borel set in $E_{n}$, then the intersections of the lines (1) with $B$ are convex linear sets and so are $\mathscr{F}_{\sigma}$-sets. Thus in this case the projection of $B$ on the plane (2) is a convex Borel set. As any projection can be split into a sequence of projections reducing the dimension one step at a time we have:

THEOREM 1. Any projection of a convex Borel set in a finite dimensional Euclidean space is a convex Borel set. 
As there is no reasonably simple proof of the Kunugui-Novikoff theorem we give a relatively simple proof of Theorem 1 .

Proof of Theorem 1. Let $B$ be a bounded convex Borel set in $E_{n}$.

We call a Borel set $V$ in $E_{n}$ vertically convex if each section of $V$ by a line of the form (1) is a linear convex set. We introduce some operations that lead from vertically convex Borel sets to vertically convex Borel sets. If $V$ is any vertically convex Borel set, let $\mathrm{O} V$ denote the set

$$
\mathrm{O} V=\bigcup_{n=1}^{\infty}\left[\left(V+\frac{1}{n} \mathbf{e}\right) \cap\left(V-\frac{1}{n} \mathbf{e}\right)\right]
$$

where

$$
\mathrm{e}=(0,0, \cdots, 0,1),
$$

and we use the standard vector addition. Then $O V$ is clearly a Borel set as the sets $V \pm(1 / n)$ e are Borel sets. Further the section of $O V$ by any line of the form (1) will be the relative interior of the corresponding linear convex set forming the intersection of the line with $V$. In particular $\mathrm{O} V$ will be vertically convex.

For each positive integer $n$, let $D_{n}$ be the operator replacing any bounded vertically convex set $V$ by the set

$$
\mathbf{D}_{n} V=V \backslash\left\{\mathbf{O} V+(-1)^{n} \frac{1}{n} \mathrm{e}\right\} \text {. }
$$

Clearly, if $V$ is a bounded vertically convex Borel set, $\mathbf{D}_{n} V$ is a Borel set. Further, the effect of $D_{n}$ is to leave unchanged any 'vertical' section of length less than or equal to $1 / n$ and to shorten any longer interval down to the length $1 / n$, leaving the interval closed at the shortened end.

We now consider the set

$$
U=\bigcap_{n=1}^{\infty} B_{n},
$$

where we take $B_{0}=B$ and

$$
B_{n}=\mathrm{D}_{n} B_{n-1}, \quad n=1,2, \cdots .
$$

As $B$ is a bounded vertically convex Borel set, it follows that the sets, $B_{1}, B_{2}, \cdots$, and $U$ are bounded vertically convex Borel sets. Those vertical sections of $B$ that consist of single points remain in all the sets $B_{1}, B_{2}, \cdots$ and $U$. Those vertical sections of $B$ that have length exceeding $1 / n$ but not exceeding $1 /(n-1)$ remain in the sets $B_{0}, B_{1}, \cdots, B_{n-1}$, are cut down to intervals, perhaps half open, 
of length $1 / n$ in $B_{n}$, and are cut down to closed intervals of length $1 / m$ in $\mathrm{B}_{m}$ for $m \geqq n+1$, and so remain as single points in $U$. Thus $U$ is a Borel set with the same projection on $x_{n}=0$ as $B$, and each vertical section of $U$ contains at most one single point. Hence, by a result of Souslin, see for example [10], the projection of $B$, being a one-to-one continuous image of the Borel set $U$, is itself Borel.

As any convex Borel set is a countable union of bounded convex Borel sets, it follows that the projection of any convex Borel set is a convex Borel set. This result extends immediately to general projections reducing the dimensionality by a finite number.

We use this result to establish

THeOREM 2. If $H, K$ are convex Borel sets in $E_{n}$, the least convex set containing $H \cup K$ is a Borel set.

Proof. Let $I$ denote the unit interval $0 \leqq x_{1} \leqq 1$ in $E_{1}$. As $H$, $K, I$ are convex Borel sets, the set

$$
H \times K \times I
$$

in $E_{2 n+1}$ is a convex Borel set. Now consider the map $\varphi$ of $E_{2 n+1}$ to itself that takes the point

$$
\mathbf{x}=\left(x_{1}, x_{2}, \cdots, x_{2 n+1}\right)
$$

to the point

$$
\begin{aligned}
& \varphi \mathrm{x}=\left(\left(1-x_{2 n+1}\right) x_{1},\left(1-x_{2 n+1}\right) x_{2}, \cdots,\left(1-x_{2 n+1}\right) x_{n},\right. \\
& \left.x_{2 n+1} x_{n+1}, x_{2 n+1} x_{n+2}, \cdots, x_{2 n+1} x_{2 n}, x_{2 n+1}\right) \text {. }
\end{aligned}
$$

When restricted to the region $S$ defined by

$$
0<x_{2 n+1}<1,
$$

the map $\varphi$ is a one-to-one and bi-continuous map of $S$ into itself. As $S$ is a Borel set in $E_{2 n+1}$ it follows that

$$
\varphi\{H \times K \times I \cap S\}
$$

is a Borel set in $E_{2 n+1}$. Now

$$
\varphi\{H \times K \times I\}=\varphi\{H \times K \times I\} \cup\{H \times\{\mathbf{o}\} \times\{0\}\} \cup\{\{\mathbf{o}\} \times K \times\{1\}\},
$$

where $\{\mathbf{o}\}$ denotes the set consisting of the origin of $E_{n}$, and $\{0\}$ is the set containing only the real number 0 and similarly $\{1\}$ is the set containing only the real number 1 . Hence

$$
\varphi\{H \times K \times I\}
$$


is a Borel set in $E_{2 n+1}$.

By standard vector algebra the set $\varphi\{H \times K \times I\}$ is convex. Further the least convex cover of $H \cup K$ is obtained from $\varphi\{H \times K \times I\}$ by application of the projection $\psi$ taking the point

$$
\mathbf{x}=\left(x_{1}, x_{2}, \cdots, x_{2 n+1}\right)
$$

to the point

$$
\psi \mathrm{x}=\left(x_{1}+x_{n+1}, \quad x_{2}+x_{n+2}, \cdots, x_{n}+x_{2 n}\right)
$$

in $E_{n}$. Thus by Theorem 1 the set

$$
\psi \varphi\{H \times K \times I\}
$$

is a convex Borel set and is the least convex set containing $H \cup K$.

REMARKS. If we knew, in addition, that $H$ and $K$ were convexly generated convex Borel sets, it would follow without difficulty that $\varphi\{H \times K \times I\}$ would be a convexly generated Borel set. If Theorem 1 could be modified to show that convexly generated Borel sets project to give convexly generated Borel sets we would be able to establish that weakly convexly generated Borel sets are necessarily convexly generated Borel sets.

3. Weakly convexly generated Borel sets. We prove the almost trivial.

THEOREM 3. The class of weakly convexly generated Borel sets is closed under the operations of taking the least convex cover of any countable union.

Proof. Let $K_{1}, K_{2}, \cdots$ be any sequence of weakly convexly generated Borel sets. We use conv. $E$ to denote the least convex set containing a given set $E$. Then, it follows by induction that the sets $H_{1}, H_{2}, \ldots$ defined by

$$
\begin{aligned}
H_{1} & =K_{1}, \\
H_{r+1} & =\text { conv. }\left\{H_{r} \cup K_{r+1}\right\}, \quad r \geqq 1,
\end{aligned}
$$

form an increasing sequence of weakly convexly generated Borel sets. Hence

$$
\bigcup_{r=1}^{\infty} H_{r}
$$

is a weakly convexly generated Borel set. Since 


$$
\text { conv. } \bigcup_{r=1}^{\infty} K_{r}=\bigcup_{r=1}^{\infty} H_{r} \text {, }
$$

this proves the closure of the class under the given operation.

4. The existence of convex-Borel-Souslin representations, It is convenient to introduce some notation. We use $\mathrm{I}$ to denote the space of all vectors

$$
\mathbf{i}=i_{1}, i_{2}, \cdots,
$$

with positive integral components. We use $\mathbf{i} \mid n$ to denote the finite sequence

$$
\text { i } \mid n=i_{1}, i_{2}, \cdots, i_{n},
$$

with the convention that $\mathbf{i} \mid 0$ is to denote a blank space on the paper where it is written.

We prove

THEOREM 4. The weakly convexly generated Borel sets have convex-Borel-representations.

Proof. By the definition of the weakly convexly generated Borel sets, it suffices to prove that the class of sets, having convex-BorelSouslin representations, contains the closed convex sets and is closed under the operations of taking the least convex cover of two sets of the system, of countable increasing union and of countable intersection.

First if $K$ is a compact convex set we may write

$$
F(\mathbf{i} \mid n)=K,
$$

for all $\mathbf{i}$ in $\mathrm{I}$ and all $n \geqq 1$. Then

$$
K=\bigcup_{\mathbf{i} \in \mathbf{I}} \bigcap_{n=1}^{\infty} F(\mathbf{i} \mid n),
$$

and it is easy to verify that this is a convex-Borel-Souslin representation for $K$.

Now suppose that $K^{(r)}, r=1,2, \cdots$ is an increasing sequence of sets each with a convex-Borel-Souslin representation. We may take the convex-Borel-Souslin representation for $K^{(r)}$ to be

$$
K^{(r)}=\bigcup_{\mathbf{i} \in \mathbf{I}} \bigcap_{n=1}^{\infty} F^{(r)}(\mathbf{i} \mid n) \text {. }
$$

Then

$$
K=\bigcup_{r=1}^{\infty} K^{(r)}
$$


is convex and has the convex-Borel-Souslin representation

$$
K=\bigcup_{i \in \mathbf{I}} \bigcap_{n=1}^{\infty} F(i \mid n)
$$

on writing

$$
F\left(2^{j_{0}-1}\left(2 j_{1}-1\right), j_{2}, j_{3}, \cdots, j_{n}\right)=F^{\left(j_{0}\right)}\left(j_{1}, j_{2}, \cdots, j_{n}\right),
$$

for all sequences $j_{0}, j_{1}, \cdots, j_{n}$ of positive integers with $n \geqq 1$.

As each closed convex set is the increasing union of a sequence of compact convex sets, it follows that each closed convex set has a convex-Borel-Souslin representation.

Now suppose that if $K^{(1)}, K^{(2)}$ have convex-Borel-Souslin representations of the form (3) with $r=1,2$. By replacing $F^{(r)}(\mathbf{i} \mid n)$ by

$$
\bigcap_{k=1}^{n} F^{(r)}(\mathbf{i} \mid k) \text {. }
$$

we obtain similar representations with the additional condition

$$
F^{(r)}(\mathbf{i} \mid 1) \supset F^{(r)}(\mathbf{i} \mid 2) \supset \cdots,
$$

for $r=1,2$ and all $i$ in $\mathrm{I}$.

For each vector $\mathbf{k}$ in $\mathbf{I}$ with

$$
k_{1}=2^{h_{0}-1}\left(2 h_{1}-1\right) \text {, }
$$

write

$$
\begin{aligned}
& \mathbf{i}(\mathbf{k})=h_{0}, k_{2}, k_{4}, k_{6}, \cdots, \\
& \mathbf{j}(\mathbf{k})=h_{1}, k_{3}, k_{5}, k_{7}, \cdots .
\end{aligned}
$$

Define convex sets

$$
K(\mathbf{k} \mid n), \quad \mathbf{k} \in \mathbf{I}, \quad n=1,2, \cdots,
$$

by taking

$$
K(\mathbf{k} \mid n)=\operatorname{conv} \cdot\left\{F^{(1)}(\mathbf{i}(\mathbf{k}) \mid n) \cup F^{(2)}(\mathbf{j}(\mathbf{k}) \mid n)\right\} .
$$

As $F^{(1)}(\mathbf{i}(\mathbf{k}) \mid n)$ and $F^{(2)}(\mathbf{j}(\mathbf{k}) \mid n)$ are compact so is $K(\mathbf{k} \mid n)$. It follows, without difficulty, that conv. $\left\{K^{(1)} \cup K^{(2)}\right\}$ has the Souslin representation

$$
\text { conv. }\left\{K^{(1)} \cup K^{(2)}\right\}=\bigcup_{\mathbf{k} \in \mathbf{I}} \bigcap_{n=1}^{\infty} K(\mathbf{k} \mid n)
$$

the set

$$
\bigcup_{\substack{\mathbf{h} \in \mathbf{I} \\ \mathbf{h}|n=\mathbf{k}| n}} \bigcap_{r=1}^{\infty} K(\mathbf{h} \mid r)
$$


taking the form

$$
\operatorname{conv} \cdot\left[\left\{\bigcup_{\substack{\mathbf{i} \in \mathbf{I} \\ \mathbf{i}|m=\mathbf{i}(\mathbf{k})| m}} \bigcap_{r=1}^{\infty} F^{(1)}(\mathbf{i} \mid r)\right\} \cup\left\{\bigcup_{\substack{\mathbf{j} \in \mathbf{I} \\ \mathbf{j}\left|m^{\prime}=\mathbf{j}(\mathbf{k})\right| m^{\prime}}} \bigcap_{r=1}^{\infty} F^{(2)}(\mathbf{j} \mid r)\right\}\right],
$$

with $m=m^{\prime}=1 / 2(n+1)$ if $n$ is odd and $m=1 / 2 n, m^{\prime}=m-1$ if $n$ is even. Thus, using Theorem 2 , the representation is a convex-BorelSouslin representation.

Finally consider the intersection of a sequence $K^{(r)}, r=1,2, \cdots$ of sets having convex-Borel-Souslin representations of the form (3) with (4) satisfied for $r=1,2, \cdots$. Following one of the standard proofs that the intersection of any sequence of Souslin sets is a Souslin set we introduce a sequence

$$
\mathbf{i}_{1}(\mathbf{k}), \mathbf{i}_{2}(\mathbf{k}), \cdots
$$

of vectors associated with any vector $\mathbf{k}$ of $\mathbf{I}$, by taking

$$
\begin{aligned}
& \mathbf{i}_{1}(\mathbf{k})=k_{1}, k_{3}, k_{5}, \cdots, \\
& \mathbf{i}_{2}(\mathbf{k})=k_{1.2}, k_{3.2}, k_{5.2}, \cdots, \\
& \mathbf{i}_{3}(\mathbf{k})=k_{1.4}, k_{3.4}, k_{5.4}, \cdots, \\
& \ldots,
\end{aligned}
$$

the general suffix being expressed as the product of an odd number with a power of 2 . We then write

$$
K(\mathbf{k} \mid n)=\bigcap_{(2 q-1) 2 h-1 \leqq n} F^{(h)}\left(\mathbf{i}_{h}(\mathbf{k}) \mid q\right) .
$$

Standard arguments show that

$$
\bigcap_{n=1}^{\infty} K^{(h)}=\bigcup_{\mathbf{k} \in \mathbf{I}} \bigcap_{n=1}^{\infty} K(\mathbf{k} \mid n),
$$

the right-hand-side yielding a Souslin representation. Further, each set

$$
\bigcup_{\substack{\mathbf{h} \in \mathbf{I} \\ \mathbf{h}|n=\mathbf{k}| n}} \bigcap_{r=1}^{\infty} K(\mathbf{h} \mid r)
$$

turns out to be the finite intersection of those sets

$$
\bigcup_{\substack{\mathbf{j} \in \mathbf{I} \\ \mathbf{j}\left|q=\mathbf{i}_{h}(\mathbf{k})\right| q}} \bigcap_{t=1}^{\infty} F^{(h)}(\mathbf{j} \mid t),
$$

with $2^{h-1}(2 q-1) \leqq n$. As these sets are convex Borel sets, the representation (5) is a convex-Borel-Souslin representation. This completes the proof. 


\section{REFERENCES}

1. P. Erdös and A. H. Stone, On the sum of Borel sets, Proc. Amer. Math. Soc. 25 (1970), 304-306.

2. V. L. Klee, Convex sets in linear spaces, Duke Math. J., 18 (1951), 443-466.

3. — Can all convex Borel set be generated in a Borelian manner within the realm of convexity? Amer. Math. Monthly, 76 (1969), 678-679.

4. K. Kunugui, Sur un probleme de M. B. Szpilrajn, Proc. Imp. Acad. Tokyo, 16 (1940), 73-78.

5. K. Kuratowski, Topology (Academic Press, New York, 1966), 487.

6. D. C. Larman, The convex Borel sets in $R^{3}$ are convexly generated, to appear in J. London Math. Soc.

7. - On the convex generation of convex Borel sets in $R^{3}$, J. London Math. Soc., (2) 1 (1969), 101-108.

8. C. A. Rogers, A linear Borel set whose difference set is not a Borel set, Bull. London Math. Soc., 1 (1969).

9. — Spaces with good Borel structures, J. London Math. Soc., (2), 2 (1970).

Received December 16, 1969. I am grateful to Dr. D. C. Larman for most valuable discussions on this topic, during which he kept me informed of his own work.

UNIVERSITY COLLEGE LONDON 


\section{PACIFIC JOURNAL OF MATHEMATICS}

\section{EDITORS}

\author{
H. SAMELSON \\ Stanford University \\ Stanford, California 94305 \\ Richard Pierce \\ University of Washington \\ Seattle, Washington 98105
}

\author{
J. DugundJI \\ Department of Mathematics \\ University of Southern California \\ Los Angeles, California 90007 \\ RICHARD ARENS \\ University of California \\ Los Angeles, California 9.0024
}

\section{ASSOCIATE EDITORS}

\begin{tabular}{|c|c|}
\hline E. F. BECKENBACH & K. YoshidA \\
\hline \multicolumn{2}{|c|}{ SUPPORTING INSTITUTIONS } \\
\hline UNIVERSITY OF BRITISH COLUMBIA & STANFORD UNIVERSITY \\
\hline CALIFORNIA INSTITUTE OF TECHNOLOGY & UNIVERSITY OF TOKYO \\
\hline UNIVERSITY OF CALIFORNIA & UNIVERSITY OF UTAH \\
\hline MONTANA STATE UNIVERSITY & WASHINGTON STATE UNIVERSITY \\
\hline UNIVERSITY OF NEVADA & UNIVERSITY OF WASHINGTON \\
\hline NEW MEXICO STATE UNIVERSITY & * \\
\hline OREGON STATE UNIVERSITY & AMERICAN MATHEMATICAL SOCIETY \\
\hline UNIVERSITY OF OREGON & CHEVRON RESEARCH CORPORATION \\
\hline OSAKA UNIVERSITY & TRW SYSTEMS \\
\hline UNIVERSITY OF SOUTHERN CALIFORNIA & NAVAL WEAPONS CENTER \\
\hline
\end{tabular}

The Supporting Institutions listed above contribute to the cost of publication of this Journal, but they are not owners or publishers and have no responsibility for its content or policies.

Mathematical papers intended for publication in the Pacific Journal of Mathematics should be in typed form or offset-reproduced, (not dittoed), double spaced with large margins. Underline Greek letters in red, German in green, and script in blue. The first paragraph or two must be capable of being used separately as a synopsis of the entire paper. The editorial "we" must not be used in the synopsis, and items of the bibliography should not be cited there unless absolutely necessary, in which case they must be identified by author and Journal, rather than by item number. Manuscripts, in duplicate if possible, may be sent to any one of the four editors. Please classify according to the scheme of Math. Rev. Index to Vol. 39. All other communications to the editors should be addressed to the managing editor, Richard Arens, University of California, Los Angeles, California, 90024.

50 reprints are provided free for each article; additional copies may be obtained at cost in multiples of 50 .

The Pacific Journal of Mathematics is published monthly. Effective with Volume 16 the price per volume (3 numbers) is $\$ 8.00$; single issues, $\$ 3.00$. Special price for current issues to individual faculty members of supporting institutions and to individual members of the American Mathematical Society: $\$ 4.00$ per volume; single issues $\$ 1.50$. Back numbers are available.

Subscriptions, orders for back numbers, and changes of address should be sent to Pacific Journal of Mathematics, 103 Highland Boulevard, Berkeley, California, 94708.

PUBLISHED BY PACIFIC JOURNAL OF MATHEMATICS, A NON-PROFIT CORPORATION

Printed at Kokusai Bunken Insatsusha (Internatıonal Academic Printing Co., Ltd.), 7-17, Fujimi 2-chome, Chiyoda-ku, Tokyo, Japan. 


\section{Pacific Journal of Mathematics}

\section{Vol. 35, No. $3 \quad$ November, 1970}

John D. Arrison and Michael Rich, On nearly commutative degree one algebras . . . 533

Bruce Alan Barnes, Algebras with minimal left ideals which are Hilbert spaces . . . . 537

Robert F. Brown, An elementary proof of the uniqueness of the fixed point index . . . 549

Ronn L. Carpenter, Principal ideals in F-algebras .................... 559

Chen Chung Chang and Yiannis (John) Nicolas Moschovakis, The Suslin-Kleene

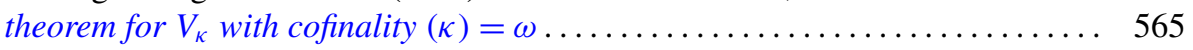

Theodore Seio Chihara, The derived set of the spectrum of a distribution

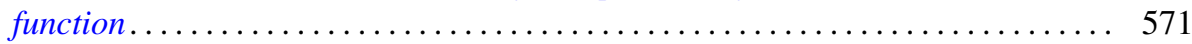

Tae Geun Cho, On the Choquet boundary for a nonclosed subspace of $C(S) \ldots \ldots \quad 575$

Richard Brian Darst, The Lebesgue decomposition, Radon-Nikodym derivative,

conditional expectation, and martingale convergence for lattices of sets .......

David E. Fields, Dimension theory in power series rings . . . . . . . . . . . .

Michael Lawrence Fredman, Congruence formulas obtained by counting

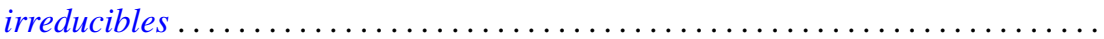

John Eric Gilbert, On the ideal structure of some algebras of analytic functions.....

G. Goss and Giovanni Viglino, Some topological properties weaker than

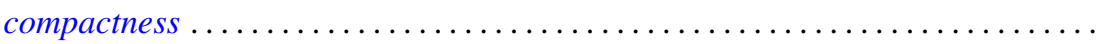

581

601

625

George Grätzer and J. Sichler, On the endomorphism semigroup (and category) of

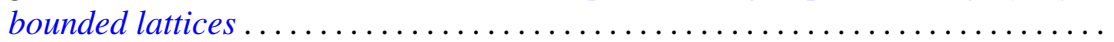

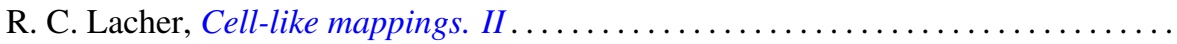

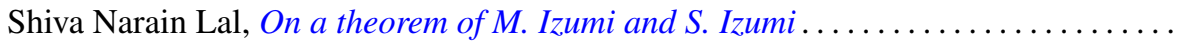

661

Howard Barrow Lambert, Differential mappings on a vector space ...............

Richard G. Levin and Takayuki Tamura, Notes on commutative power joined

semigroups.

Robert Edward Lewand and Kevin Mor McCrimmon, Macdonald's theorem for quadratic Jordan algebras.

J. A. Marti, On some types of completeness in topological vector spaces ....

Walter J. Meyer, Characterization of the Steiner point

717

Saad H. Mohamed, Rings whose homomorphic images are $q$-rings ...

727

Thomas V. O'Brien and William Lawrence Reddy, Each compact orientable surface

of positive genus admits an expansive homeomorphism ...

737

Robert James Plemmons and M. T. West, On the semigroup of binary relations...

743

Calvin R. Putnam, Unbounded inverses of hyponormal operator . .

755

William T. Reid, Some remarks on special disconjugacy criteria for differential

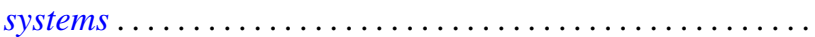

C. Ambrose Rogers, The convex generation of convex Borel sets in euclidean

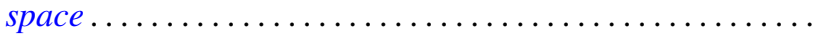

S. Saran, A general theorem for bilinear generating functions .

S. W. Smith, Cone relationships of biorthogonal systems ......

Wolmer Vasconcelos, On commutative endomorphism rings ....

795

Vernon Emil Zander, Products of finitely additive set functions from Orlicz

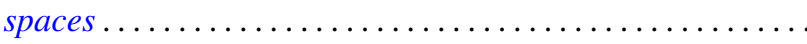

G. Sankaranarayanan and C. Suyambulingom, Correction to: "Some renewal

theorems concerning a sequence of correlated random variables" .

Joseph Zaks, Correction to: "Trivially extending decompositions of $E^{n}$ ”....... 805

Dong Hoon Lee, Correction to: "The adjoint group of Lie groups" ............ 805

James Edward Ward, Correction to: "Two-groups and Jordan algebras". 